\title{
FISH analysis helps identify low-level mosaicism in Ullrich-Turner syndrome patients
}

Anne Wiktor, BS CLSp(CG) and Daniel L. Van Dyke, PhD

\begin{abstract}
Purpose: To search for $X$ or $Y$ chromosome mosaicism in 45,X individuals using fluorescent in situ hybridization (FISH). Methods: From our series of 53 Ullrich-Turner syndrome patients, we used interphase FISH to evaluate the 19 who had an apparently nonmosaic 45,X karyotype with G-banding. Results: Of those 19 patients, mosaicism was detected in seven (37\%), five patients had an XX line, one had a monocentric isochromosome $X$, and one had a dicentric isochromosome $X$. No $Y$ chromosome mosaic was identified. Conclusion: FISH analysis is a sensitive and cost-effective adjunct to karyotype analysis to identify sex chromosome mosaicism in UTS. Genet Med 2004: 6(3):132-135.
\end{abstract}

Key Words: Turner syndrome, $X$ chromosome monosomy, gonadoblastoma, chromosomal mosaicism

The incidence of Ullrich-Turner syndrome (UTS) is approximately one in 3000 newborn girls and is associated with an apparently nonmosaic 45 , X karyotype in many of these patients. ${ }^{1}$ Fewer than $15 \%$ of UTS patients appear to have mosaicism with a 46,XY cell population or a Y chromosome rearrangement, and $30-50 \%$ are mosaic with a second $\mathrm{X}$ or a structurally abnormal X. Up to $18 \%$ of patients have a small marker chromosome in some cells, the origin of which can be identified by FISH or other methods. ${ }^{2}$ The mosaic status of the remaining UTS patients remains uncertain but of clinical interest because if they do have cells with a Y chromosome, they may have an increased risk of gonadoblastoma. The American College of Medical Genetics recommends cytogenetic analysis of 30 metaphase cells in cases studied to rule out sex chromosome mosaicism. ${ }^{3}$ This analysis can identify $10 \%$ mosaicism with a confidence level of $95 \%$, but a more sensitive level of detection would require costly analysis of many more metaphase cells. PCR-based assays can be used to identify low-level mosaicism but has limitations ${ }^{4-7}$ including a high rate of falsepositive results with nested PCR. ${ }^{8,9}$ In this study, we report the results of karyotype and FISH analysis in 53 patients with monosomy $\mathrm{X}$ or monosomy $\mathrm{X}$ mosaicism.

\section{MATERIALS AND METHODS}

Between January 1997 and August 2003, we studied 53 samples submitted for karyotype analysis based on phenotypic features suggestive of UTS in which the karyotype results included a 45,X cell line. We excluded females suspected of having UTS who had a nonmosaic 46,XX karyotype. Several blood samples

Henry Ford Hospital, Detroit, Michigan.

Anne Wiktor, Henry Ford Hospital, 2799 West Grand Boulevard, Detroit, MI 48202.

Received: November 18, 2003.

Accepted: January 14, 2004.

DOI: 10.1097/01.GIM.0000127270.49902.56 were sent to confirm a prenatal diagnosis of UTS were included.

G-banded metaphase analysis from PHA-stimulated lymphocyte cultures was performed. A minimum of 30 metaphase cells was analyzed in cases with a nonmosaic 45 , X karyotype, or a minimum of 20 metaphase cells when mosaicism was identified by the 20th cell.

FISH testing was performed on all 19 cases with an apparently nonmosaic 45 , X karyotype. Using centromere probes for the $\mathrm{X}$ (DXZ1) and Y chromosomes (DYZ3) (Vysis, Inc., Downer's Grove, IL), a minimum of 200 interphase cells were scored. A male control was hybridized concurrently with each sample and the intensity of the $\mathrm{Y}$ signal was used to discriminate between a true " $Y$ " signal and an artifact signal. If the initial analysis was equivocal (one cell with both $\mathrm{X}$ and $\mathrm{Y}$ signals), an additional 300 cells were analyzed. Cases demonstrating one XX signal (of 200 cells) or one XY signal (of $>500$ cells) were considered nonmosaic, whereas those with $>1 \% \mathrm{XX}$ cells were identified as true mosaics. If mosaicism was identified by FISH in $>5 \%$ of cells, additional metaphase cells were analyzed to determine if the second $\mathrm{X}$ signal represented a structurally normal X chromosome.

\section{RESULTS}

We reviewed our cytogenetic findings on 53 females with a 45 , X karyotype as the sole abnormality or as part of a more complex karyotype (Table 1). Nineteen patients (36\%) revealed a 45,X karyotype, 16 (36\%) exhibited numerical mosaicism involving the $\mathrm{X}$ chromosome, four (7\%) patients had an isochromosome X, two (4\%) had a deletion of the short arm of the $\mathrm{X}$ chromosome, and five (9\%) had a ring $\mathrm{X}$ chromosome. Mosaicism with a structurally normal $Y$ chromosome was seen in three (5\%) cases, two (4\%) patients had a dicentric $\mathrm{Y}$, and one $(2 \%)$ case had mosaicism with a small marker chromosome that was determined by FISH analysis to be derived from the $\mathrm{Y}$ chromosome. One (2\%) patient showed mosaicism for a 
Table 1

Cytogenetic analysis data of 53 female patients with UTS and an abnormal karyotype

\begin{tabular}{lccc}
\hline Karyotype result & No. patients & \% of patients & \% of 45, cells $^{a}$ \\
\hline $45, \mathrm{X}^{a}$ & 19 & 36 & \\
$45, \mathrm{X} / 46, \mathrm{XX}$ & 10 & 18 & $6-60$ \\
$45, \mathrm{X} / 46, \mathrm{XX} / 47, \mathrm{XXX}$ & 3 & 6 & $5-78$ \\
$45, \mathrm{X} / 47, \mathrm{XXX}$ & 3 & 6 & $13-98$ \\
$45, \mathrm{X} / 46, \mathrm{X}, \operatorname{del}(\mathrm{X})(\mathrm{p} 11.2)$ & 1 & 2 & 3 \\
$45, \mathrm{X} / 46, \mathrm{X}, \mathrm{del}(\mathrm{X})(\mathrm{q} 21.1)$ & 1 & 2 & 74 \\
$45, \mathrm{X} / 46, \mathrm{X}, \mathrm{idict}(\mathrm{X})(\mathrm{q} 25)$ & 1 & 2 & 90 \\
$45, \mathrm{X} / 46, \mathrm{X}, \mathrm{i}(\mathrm{X})(\mathrm{q} 10)$ & 3 & 5 & $20-60$ \\
$45, \mathrm{X} / 46, \mathrm{X}, \mathrm{r}(\mathrm{X})(\mathrm{p} 22.3 \mathrm{q} 23)$ & 1 & 2 & 50 \\
$45, \mathrm{X} / 46, \mathrm{X}, \mathrm{r}(\mathrm{X})(\mathrm{p} 22.3 \mathrm{q} 24)$ & 1 & 2 & 60 \\
$45, \mathrm{X} / 46, \mathrm{X}, \mathrm{r}(\mathrm{X})(\mathrm{p} 11 \mathrm{q} 11)$ & 3 & 5 & $50-97$ \\
$45, \mathrm{X} / 46, \mathrm{XY}$ & 2 & 4 & $10-80$ \\
$45, \mathrm{X} / 46, \mathrm{XY} / 47, \mathrm{XYY}$ & 1 & 2 & 20 \\
$45, \mathrm{X} / 46, \mathrm{idic}(\mathrm{Y})(\mathrm{q} 11.2)$ & 2 & 4 & $10-63$ \\
$45, \mathrm{X} / 46, \mathrm{X}, \mathrm{r}(\mathrm{Y})$ & 1 & 2 & 98 \\
$45, \mathrm{X} / 46, \mathrm{X},+$ mar ${ }^{b}$ & 1 & 2 & 96 \\
\hline
\end{tabular}

${ }^{a}$ Based on a minimum of 30 metaphase cells.

${ }^{b}$ Marker was not X- or Y-derived based on FISH analysis.

marker chromosome that was derived from neither the X nor Y chromosomes.

The interphase FISH analysis identified chromosomal mosaicism in 7 of the 19 patients (37\%) with an apparently nonmosaic 45, X karyotype, using centromere probes for the $\mathrm{X}$ and Y chromosomes (Table 2). None of the FISH studies revealed the presence of a Y-derived chromosome. Cases no. 4, 6, 14, 17, and 18 demonstrated a second cell line with two X chromosome signals. In the course of the interphase analysis of case no. 8 , we identified metaphase cells with a normal X chromosome and a monocentric isochromosome Xq (Fig. 1a). Case no. 7 showed a mixture of interphase cells with one and three $\mathrm{X}$ chromosome signals, with two of the three $\mathrm{X}$ signals in close proximity, suggesting the presence of a dicentric $\mathrm{X}$ chromosome (Fig. 1b). The abnormal chromosome was identified in 2 of 55 additional G-banded metaphase cells, and confirmed to be a idic(X)(q22) (Fig. 1c). The FISH results for 12 of the 19 cases were uniformly monosomy $\mathrm{X}$.

\section{DISCUSSION}

Because the phenotypic expression and clinical management of patients with UTS is dependent upon the karyotype result, identification of sex chromosome mosaicism plays a key role in prognosis and treatment. Patients with a Y or Y-derived chromosome identified by routine G-banding analysis have a $15-25 \%$ increased risk of developing gonadoblastoma. There-
Table 2

Interphase FISH analysis results for 19 patients with a 45,X G-banded karyotype $^{a}$

\begin{tabular}{|c|c|c|c|c|c|c|}
\hline \multirow[b]{2}{*}{ Case no. } & \multicolumn{3}{|c|}{$\mathrm{X}$ signals } & \multicolumn{3}{|c|}{ Y signals } \\
\hline & 1 & 2 & 3 & 1 & 2 & 3 \\
\hline 1 & 200 & & & & & \\
\hline 2 & 200 & & & & & \\
\hline 3 & 200 & & & & & \\
\hline 4 & 200 & 10 & & & & \\
\hline 5 & 600 & & & 1 & & \\
\hline 6 & 200 & 2 & & & & \\
\hline 7 & 200 & & 19 & & & \\
\hline $8^{b}$ & 500 & 19 & & 1 & & \\
\hline 9 & 500 & & & & & \\
\hline 10 & 300 & 1 & & & & \\
\hline 11 & 200 & & & & & \\
\hline 12 & 200 & & & & & \\
\hline 13 & 200 & & & & & \\
\hline 14 & 200 & 4 & & & & \\
\hline 15 & 500 & & & & & \\
\hline 16 & 500 & & & 1 & & \\
\hline 17 & 200 & 6 & & & & \\
\hline 18 & 200 & 2 & & & & \\
\hline 19 & 200 & 1 & & & & \\
\hline
\end{tabular}

$\overline{{ }^{a} \text { Cases with one cell having an XY signal (of } 500-1000 \text { cells) or one XX signal }}$ (of 200 cells) were regarded as nonmosaic cases.

${ }^{b} \mathrm{FISH}$ analysis revealed several metaphase cells with one normal $\mathrm{X}$ and one isochromosome $\mathrm{X}$.

fore, identification of low-level Y chromosome mosaicism is potentially also clinically important.

A 45 ,X karyotype has been identified in roughly $50 \%$ of UTS patients, with $15 \%$ showing mosaicism for a structurally normal XX cell line, $20 \%$ with an isochromosome Xq, $10 \%$ with $\mathrm{X} / \mathrm{XY}$ mosaicism, and the remainder with $\mathrm{X}$ or $\mathrm{Y}$ rearrangements. ${ }^{1}$

FISH testing has been used as an adjunct to karyotype analysis in prenatal and postnatal analysis of samples with a $45, \mathrm{X}$ karyotype. In a study of 53 women, by Hanson et al., ${ }^{10}$ FISH identified a Y-derived chromosome in eight cases as compared to six by cytogenetics, and X-chromosome mosaicism by FISH in 37 cases versus 24 by karyotype analysis.

Using FISH analysis, Abulhasan et al., ${ }^{11}$ confirmed karyotype results in 8 of $22(36 \%)$ cases, identified a third cell line in $14(64 \%)$ cases, determined the origin of five $(23 \%)$ marker chromosome as $\mathrm{X}$-derived, and clarified a $\operatorname{del}(\mathrm{Y})$ in two (9\%) cases with a 45,X/46,XY karyotype. The identification of a nonmosaic 45 , X result in a prenatal sample requires clinical correlation. In two cases, a 45,X karyotype result was reported and ultrasound examination identified male genitalia. Reevaluation of 

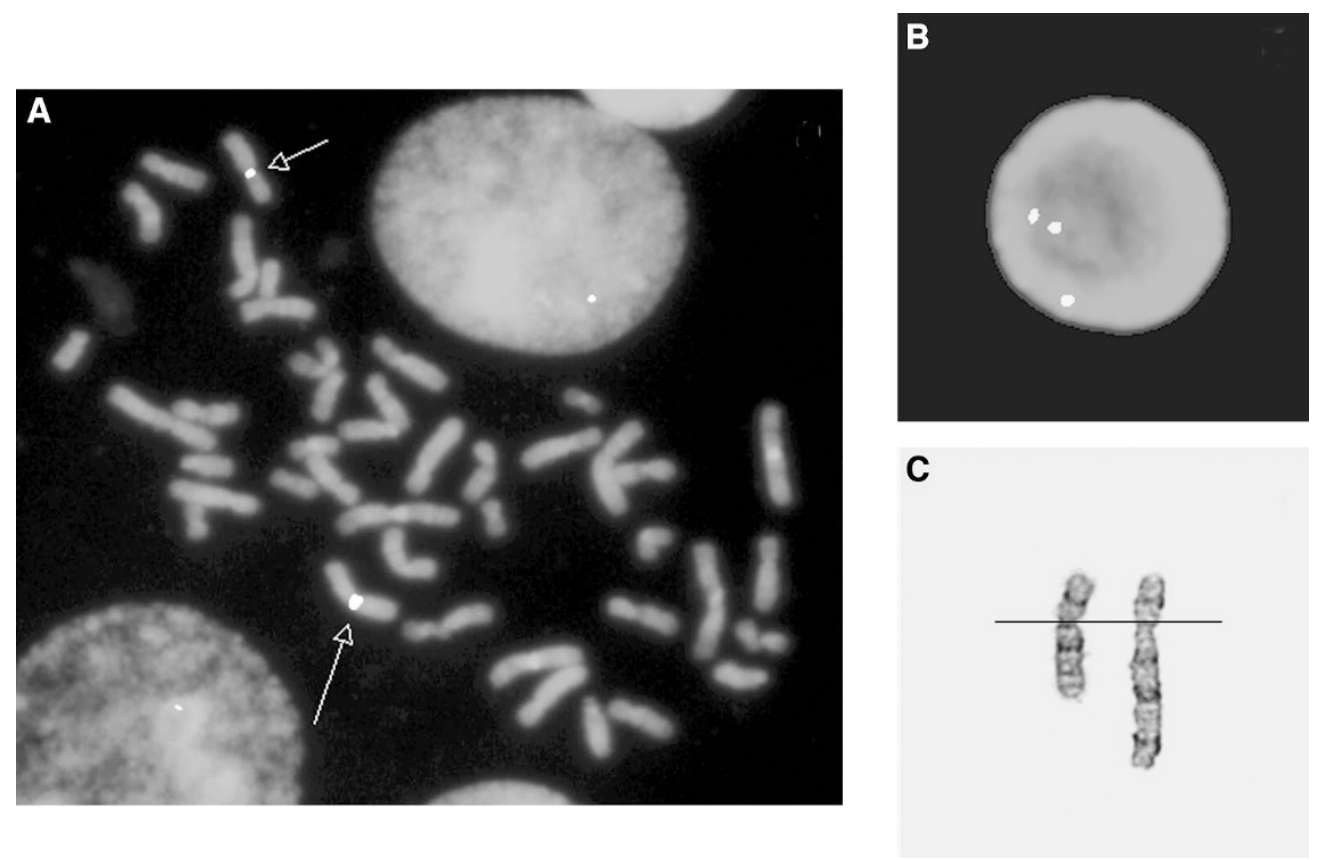

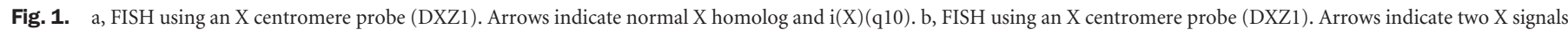
in close proximity suggestive of a dicentric X chromosome. c, Partial karyotype of normal X and dicentric X chromosomes.

all available metaphases in both cases identified marker chromosomes that FISH analysis determined to be Y-derived. ${ }^{12}$

Two studies compared methods to identify sex chromosome mosaicism in UTS patients but neither demonstrated that PCR analysis identified XY mosaicism that would not have been detected using karyotype and FISH analysis. Alvarez-Nava et al. ${ }^{13}$ identified $4 / 52$ patients with Y-chromosome material. Two patients had a 45,X/46,XY karyotype, and one had a Yderived marker chromosome identified by FISH. Peripheral blood analysis on the fourth patient identified a 45,X[50] karyotype, but Y-sequences were evident by DNA analysis; FISH analysis of gonadal tissue identified a Y-derived marker chromosome that was confirmed by PCR testing. It is possible that had FISH analysis been performed on the blood sample, $\mathrm{X} / \mathrm{XY}$ mosaicism could have been identified. In a study of 41 patients with Turner syndrome, FISH identified an XX cell population in $4 / 16$ patients who were initially identified as having a nonmosaic $45, \mathrm{X}$ karyotype. ${ }^{4}$ Two patients were found to have $\mathrm{Y}$ chromosomal material by FISH and PCR studies; one had a 45,X/46,XY karyotype and the other had a $\operatorname{dic}(\mathrm{Yp})$ that was initially described as a $\operatorname{der}(\mathrm{X})$.

In a study comparing only cytogenetic analysis and PCR studies, DYZ3 DNA sequences were apparent after the first round of PCR in 4/122 patients, ${ }^{8}$ all four carried a marker chromosome whose origin, in all likelihood, could have been identified by FISH analysis. Analysis using nested PCR testing identified Y-sequences in 25\% of these patients and in 14/100 (14\%) of the normal female controls. Although highly sensitive in its approach, nested PCR is not a reliable method for analysis in UTS patients. This high rate of false-positive detection could lead to unnecessary surgery for some patients. In addition, DNA testing requires another patient specimen once a karyotype result has been obtained. If a more thorough analysis is required to exclude mosaicism, analysis of a second tissue type using cytogenetics or FISH analysis may prove more informative.

All of the PCR studies described included the use of the SRY gene as one of the Y-markers, and although it is associated with male gonadal dysfunction, the gene responsible for gonadoblastoma has not yet been isolated. There is an increased risk of gonadoblastoma only if pericentromeric fragments or the entire $\mathrm{Y}$ chromosome are present, both of which can be identified using a Y-centromere FISH probe.

We now use FISH analysis as an adjunct to cytogenetic analysis in cases where a 45,X karyotype is identified. Analysis using $\mathrm{X}$ and $\mathrm{Y}$ centromere probes provides a sensitive, specific, rapid, and informative means of identifying low level $\mathrm{X}$ and $\mathrm{Y}$ mosaicism in UTS patients. Analysis can be performed using the same preparations used for cytogenetic analysis and most cytogenetics laboratories are equipped to perform FISH testing. Although interphase analysis of 200 cells provides a confidence level of $95 \%$ at detecting a cell line occurring at $2 \%$, additional cells may be scored if the results are equivocal. ${ }^{14}$ Analysis of 500 cells would theoretically identify $1 \%$ mosaicism with 0.99 confidence.

FISH analysis has allowed us to identify seven patients with a sex chromosome mosaicism among 19 cases $(37 \%)$ where cytogenetics analysis yielded only a single $45, \mathrm{X}$ cell line. We regard the identification of a cell population with a second $\mathrm{X}$ chromosome as sufficient to exclude, with a high degree of 
confidence, the presence of a Y-bearing cell population in that patient. FISH analysis using X and Y probes can successfully identify low level sex chromosome mosaicism in many patients with a 45,X G-banded karyotype and when detected can play a key role in patient care and management. Because 45,X/46,XX females have a lower risk of gonadoblastoma and a more moderate expression of Turner syndrome features, finding a second $\mathrm{X}$ cell line aids in patient counseling and management. Of greater clinical significance is the identification and follow-up when $\mathrm{X} / \mathrm{XY}$ mosaicism is confirmed. When used as an adjunct to conventional cytogenetic analysis, FISH analysis using $\mathrm{X}$ and $\mathrm{Y}$ centromere probes provides a sensitive, cost-effective, and readily available technique to identify sex chromosome mosaicism.

\section{References}

1. Van Dyke DL, Wiktor A. Clinical cytogenetics, In: McClatchey KD, editor. Clinical laboratory medicine. Baltimore: Williams \& Wilkins, 2002:589-635.

2. Held KR, Kerber S, Kaminsky E, Singh S, Goetz P, Seemanova E et al. Mosaicism in $45, \mathrm{X}$ Turner syndrome: Does survival in early pregnancy depend on the presence of two sex chromosomes? Hum Genet 1992;88:299-294.

3. American College of Medical Genetics: Standards and Guidelines for Clinical Genetic Laboratories. Available at: http://www.acmg.net/resources/s-g/s-g-yes-no.asp. Accessed: January 5, 2004.
4. Fernandez R, Garcia-Doval S, Costoya S, Pasaro E. Analysis of sex chromosome aneuploidy in 41 patients with Turner syndrome: a study of "hidden" mosaicism. Hum Genet 1996;58:210-208.

5. Yorifuji T, Muroi J, Kawai M, Sasaki H, Momoi T, Frosh K. PCR-based detection of mosaicism in Turner syndrome patients. Hum Genet 1997;99:62-65.

6. Lopez M, Canto P, Aguinaga M, Torres L, Cervantes A, Alfaro G et al. Frequency of Y chromosomal material in Mexican patients with Ullrich-Turner syndrome. Am J Med Genet 1998;760:120-124.

7. Hall JG. Detection of Y-specific sequences in patients with Turner syndrome. Am J Med Genet 2002;113:114.

8. Nishi M, Domenice S, Medeiros MA, Mendonca BB, Billerbeck AEC. Detection of Y-specific sequences in 122 patients with Turner syndrome: Nested PCR is not a reliable method. Am J Med Genet 2002;107:209-305.

9. Nishi M, Domenice S, Medeiros MA, Mendonca B, Billerbeck AEC. Reply to correspondence from Hall: "Detection of Y-specific sequences on patients with Turner syndrome”. Am J Med Genet 2002;113:115.

10. Hanson L, Bryman I, Barrenas ML, Janson PO, Wahlstrom J, Alberttson-Wikland K et al. Genetic analysis of mosaicism in 53 women with Turner syndrome. Hereditas 2001;134:153-159.

11. Abulhasan SJ, Tayel SM, Al-Awadi SA. Mosaic Turner syndrome: Cytogenetics vs FISH. Ann Hum Genet 1999;63:199-206.

12. Huang B, Thangavelu M, Bhatt S, Sandlin CJ, Wang Shengbiao. Prenatal diagnosis of 45,X and 45,X mosaicism: The need for thorough cytogenetic and clinical evaluations. Prenat Diagn 2002;22:105-110.

13. Alvarez-Nava F, Soto M, Sanchez MA, Fernandez E, Lanes R. Molecular analysis in Turner syndrome. J Pediatr 2003;142:336-340.

14. Hook EB. Exclusion of chromosomal mosaicism: tables of $90 \%, 95 \%$, and $99 \%$ confidence limits and comments on use. Am J Med Genet 1997;29:94-97. 